\title{
Gene Expression Profile in Delay Graft Function: Inflammatory Markers Are Associated with Recipient and Donor Risk Factors
}

\author{
Diego Guerrieri, ${ }^{1}$ Luis Re, ${ }^{2}$ Jorgelina Petroni, ${ }^{2}$ Nella Ambrosi, ${ }^{1}$ Roxana E. Pilotti, \\ H. Eduardo Chuluyan, ${ }^{1}$ Domingo Casadei, ${ }^{2}$ and Claudio Incardona ${ }^{3}$ \\ ${ }^{1}$ CEFYBO-School of Medicine, University of Buenos Aires, Paraguay 2155, 16th Floor, C1121ABG Buenos Aires, Argentina \\ ${ }^{2}$ Instituto de Nefrología de Buenos Aires, Cabello 3889, C1425APQ Buenos Aires, Argentina \\ ${ }^{3}$ GADOR S.A., Darwin 429, C1414CUH Buenos Aires, Argentina
}

Correspondence should be addressed to Claudio Incardona; incardona@gador.com.ar

Received 30 September 2013; Revised 19 December 2013; Accepted 15 January 2014; Published 15 May 2014

Academic Editor: Simi Ali

Copyright (C) 2014 Diego Guerrieri et al. This is an open access article distributed under the Creative Commons Attribution License, which permits unrestricted use, distribution, and reproduction in any medium, provided the original work is properly cited.

\begin{abstract}
Background. Delayed graft function (DGF) remains an important problem after kidney transplantation and reduced long-term graft survival of the transplanted organ. The aim of the present study was to determine if the development of DGF was associated with a specific pattern of inflammatory gene expression in expanded criteria of deceased donor kidney transplantation. Also, we explored the presence of correlations between DGF risk factors and the profile that was found. Methods. Seven days after kidney transplant, a cDNA microarray was performed on biopsies of graft from patients with and without DGF. Data was confirmed by real-time PCR. Correlations were performed between inflammatory gene expression and clinical risk factors. Results. From a total of 84 genes analyzed, 58 genes were upregulated while only 1 gene was downregulated in patients with DGF compared with no DGF $(P=0.01)$. The most relevant genes fold changes observed was IFNA1, IL-10, IL-1F7, IL-1R1, HMOX-1, and TGF- $\beta$. The results were confirmed for IFNA1, IL-1R1, HMOX-1 and TGF- $\beta$. A correlation was observed between TGF- $\beta$, donor age, and preablation creatinine, but not body mass index (BMI). Also, TGF- $\beta$ showed an association with recipient age, while IFNA1 correlated with recipient BMI. Furthermore, TGF- $\beta$, IFNA1 and HMOX-1 correlated with several posttransplant kidney function markers, such as diuresis, ultrasound Doppler, and glycemia. Conclusions. Overall, the present study shows that DGF is associated with inflammatory markers, which are correlated with donor and recipient DGF risk factors.
\end{abstract}

\section{Introduction}

Delayed graft function (DGF) is a frequent event after kidney transplantation that strongly correlates with a lower graft survival rate [1]. Although there are several different definitions among transplant centers and in the literature [2], the most accepted definition of DGF is the need for dialysis within one week of transplantation. The reported incidence of DGF varies from 3.4\% in living donor transplants to $31.2 \%$ in expanded criteria or $37.1 \%$ in donation after cardiac death donors [3]. However, the incidence is much higher in our Center (unpublished data) and in Latin-American Centers [4].

DGF is an independent risk factor for decreased graft survival. In the long-term, patients with DGF had a $49 \%$ pooled incidence of acute rejection compared to $35 \%$ incidence in
non-DGF patients [1]. Several factors have been ascribed for the DGF occurrence, such as donor, recipient, and transplant procedural factors [5]. Among the first factors, increased age, hypertension, creatinine clearance, vascular sclerosis, weight, female gender, and nontraumatic death have been described. The recipient related factors are the presence of a sensitization state, the ethnicity, proinflammatory cytokines, and the mean arterial pressure.

Based on the strong association between the occurrence of DGF and the risk of acute rejection, great effort has been done to understand the pathogenesis, to identify the risk factors, and to find therapies that tend to diminish the incidence of DGF. Thus, several immunologic factors and coagulant mechanisms have been described that influence the development of DGF [6-8]. However, the cold ischemia time (CIT) seems to be one of the most important factors 
TABLE 1: Inclusion and exclusion criteria.

\begin{tabular}{ll}
\hline Inclusion criteria & Exclusion criteria \\
\hline Donors & Donors \\
$>60$ years old or between 50 and 59 years who fulfilled at least 2 of the & (i) IV drugs abuse \\
following criteria. & (ii) HIV positive \\
$\begin{array}{l}\text { (i) History of hypertension } \\
\text { (ii) Stroke as cause of death }\end{array}$ & (iii) Kidneys from standard donors \\
(iii) Preablation $\mathrm{C}$ $>1.5 \mathrm{mg} / \mathrm{dL}$ & \\
Recipients & Recipients \\
(i) First disease donor kidney transplant & (i) Diabetes mellitus \\
(ii) $>18$ years & (ii) Chronic use of steroids \\
(iii) Signed informed consent & (iii) Pregnant women/lactancy period \\
(iv) Panel reactive antibody $<20 \%$ & (iv) History of cancer or linfoproliferative disorder \\
\hline
\end{tabular}

that influence the appearance of DGF $[9,10]$. Unfortunately, in our country, the CIT is very high, that is, more than 24 hours. This is in agreement with the 75\% incidence of DGF in our center. Therefore, the correct identification of the factors that influence DGF, it would benefit understanding the mechanisms responsible for the phenomenon.

In this study, we used a strategy to identify the influence that donor and recipient factors have on the inflammatory mechanisms of the DGF. We performed a microarray-based gene expression analysis and we examined the inflammatory markers on kidney biopsies of patients with and without DGF. Once the inflammatory markers were identified, correlations were performed with different donor and recipient DGF risk factors. We found that up- and downmodulated inflammatory markers were differentially correlated with singular donor and recipient risk factors.

\section{Materials and Methods}

2.1. Patients and Biopsies. Thirty four kidney transplanted patients were enrolled for these studies after giving written informed consent according to the Declarations of Helsinki. The clinical and research activities being reported are consistent with the Principles of the Declaration of Istanbul as outlined in the "Declaration of Istanbul on Organ Trafficking and Transplant Tourism”. Biopsies were obtained 7 days after transplant between December 2008 and June 2010. This study was approved by an Institutional Review Board.

Biopsies were obtained under ultrasound guidance by spring-loaded needles (ASAP Automatic Biopsy, Microvasive, Watertown, MA). Patients were grouped according to the presence of DGF. Posttransplant hemodialysis requirement was used to define DGF. Table 1 shows the inclusion and exclusion criteria for patients included in this study and Table 2 shows the clinical characteristics of the patients enrolled for this study. All patients were treated with (i) induction therapy of thymoglobulin (7-14 days) and metilprednisolone (500 mg i.v.); (ii) maintenance immunosuppression with sirolimus $(8-12 \mathrm{ng} / \mathrm{mL})$, mycophenolate sodium (1440 mg), and prednisone ( $4 \mathrm{mg} /$ day); (iii) prophylactic treatment of Ganciclovir IV (GCV-iv) $5 \mathrm{mg} / \mathrm{kg} /$ day or Valganciclovir (VGCV) $900 \mathrm{mg} /$ day and trimethoprimsulphamethoxazole (TMP-SMX).
2.2. Real-Time PCR Microarray Analysis. RNA was isolated by a phenol-based method from kidney biopsies by homogenization in $5 \mathrm{~mL}$ of TRIzol (Invitrogen, Carlsbad, CA). RNA was cleaned up with SABiosciences $\mathrm{RT}^{2}$-qPCR-Grade RNA isolation kit. The concentration and purity of RNA were determined by measuring the absorbance in a spectrophotometer. Sample dilutions were measured in $10 \mathrm{mM}$ Tris at $\mathrm{pH}$ 8. Absorbance A260/A230 ratio was greater than 1.7 and A260/A280 was greater than 2.0 in all samples analyzed. Also an aliquot of each RNA sample was run on a denaturing agarose gel and sharp bands were present for both the $18 \mathrm{~S}$ and $28 \mathrm{~S}$ ribosomal RNA. Samples were discarded if signals of RNA degradation were observed in the agarose gel such as smearing or shoulders on the RNA peaks. RNA samples $(1 \mu \mathrm{g})$ were reverse-transcribed into cDNAs using a firststrand cDNA RT kit (SABioscience, CA). Then, samples were analyzed according to the manufacturer's recommendations using the "Innate \& Adaptive Immune Responses" array in conjunction with the $\mathrm{RT}^{2}$ Profiler PCR Array System from SuperArray Bioscience (catalog number: PAHS-052Z, Frederick, MD). A total of 84 inflammatory related genes were examined (see Table 1 in Supplementary Material available online at http://dx.doi.org/10.1155/2014/167361). The array was initially performed with 16 RNA from kidney biopsies samples. For this, real-time PCR was performed using a 96well format PCR array and an Applied Biosystems 7500 realtime PCR unit. Primers for all genes for real-time PCR of the microarray analysis had been pretested and confirmed by the manufacturer. Assay includes positive and negative controls as well as three sets of housekeeping genes for normalization purposes. Analysis of real-time PCR results is based on the $\Delta \Delta \mathrm{Ct}$ method with normalization of the raw data to housekeeping genes. Data were analyzed using the web-based PCR array data analysis software (SABiosciences). A 2-fold cut off threshold was used to define up or downmodulation of the genes analyzed.

2.3. Real-Time PCR. The result of the microarray was analyzed for confirmation by using a SYBR Green-based realtime PCR. Briefly, RNA samples from 11 no DGF and 23 DGF patients were tested for IL-1R1, IL-10, IL-1F7, IFNA1, HMOX1 , and TGF- $\beta$ gene expression using the qPCR SuperMix Universal (Invitrogen, CA). Reaction solutions were prepared 
TABLE 2: Characteristics of renal transplant patients.

\begin{tabular}{|c|c|c|c|}
\hline & $\begin{array}{c}\text { Group } 1 \\
(\mathrm{DGF}, n=23)\end{array}$ & $\begin{array}{c}\text { Group } 2 \\
\text { (No DGF, } n=11)\end{array}$ & $P$ values \\
\hline Recipient age (years) & $54.7 \pm 8.2$ & $50.1 \pm 16.1$ & 0.27 \\
\hline Recipient BMI & $25.7 \pm 4.3$ & $23.5 \pm 1.3$ & 0.10 \\
\hline Time of dialysis (years) & $4.8 \pm 3.9$ & $5.67 \pm 2.7$ & 0.51 \\
\hline $\operatorname{HLA}$ MM (A, B, DR) & $3 \pm 1.7$ & $3.4 \pm 1.1$ & 0.48 \\
\hline CIT (hours) & $25.2 \pm 3$ & $23.9 \pm 5.5$ & 0.37 \\
\hline Donor age (years) & $56.4 \pm 4.8$ & $56.8 \pm 4.6$ & 0.81 \\
\hline Donor BMI & $26.9 \pm 4.2$ & $27.9 \pm 5.3$ & 0.55 \\
\hline Preablation creatinine $(\mathrm{mg} / \mathrm{dL})$ & $1.8 \pm 0.7$ & $1.3 \pm 0.3$ & 0.03 \\
\hline Day 1 diuresis (L) & $0.37 \pm 0.5$ & $2.7 \pm 2.5$ & 0.0001 \\
\hline Day 1 Eco Doppler & $0.81 \pm 0.6$ & $0.73 \pm 0.09$ & 0.66 \\
\hline Day 1 uremia (mg/dL) & $126.5 \pm 27.4$ & $119.1 \pm 26.9$ & 0.46 \\
\hline Day 1 creatinine $(\mathrm{mg} / \mathrm{dL})$ & $7.62 \pm 2$ & $9.08 \pm 1.5$ & 0.039 \\
\hline Day 1 serum $\mathrm{Na}^{+}(\mathrm{mEq} / \mathrm{L})$ & $136.4 \pm 3$ & $135.2 \pm 2.8$ & 0.27 \\
\hline Day 2 diuresis (L) & $0.45 \pm 0.56$ & $3.2 \pm 2.3$ & 0.0001 \\
\hline Day 2 uremia (mg/dL) & $142.9 \pm 28$ & $137.9 \pm 31.2$ & 0.64 \\
\hline Day 2 creatinine (mg/dL) & $8.05 \pm 2.2$ & $8.52 \pm 1.1$ & 0.52 \\
\hline Day 2 serum $\mathrm{Na}^{+}(\mathrm{mEq} / \mathrm{L})$ & $135.7 \pm 2.7$ & $136.2 \pm 2.4$ & 0.60 \\
\hline
\end{tabular}

BMI: body mass index; HLA MM: human leukocyte antigen mismatch.

using reagents from the one-step SYBR Green Quantitative RT-PCR kit (Invitrogen, CA) combined with $0.25 \mu \mathrm{M}$ of each primer and $1 \mu \mathrm{g}$ of total RNA. The settings for the PCR instrument were as follows: $42^{\circ} \mathrm{C}$ for $30 \mathrm{~min}, 94^{\circ} \mathrm{C}$ for $2 \mathrm{~min}$, and 40 cycles of $95^{\circ} \mathrm{C}$ for $15 \mathrm{~s}$ followed by $60^{\circ} \mathrm{C}$ for $1 \mathrm{~min}$. Fluorescent signals were monitored sequentially for each sample tube once per cycle at the end of the elongation step. The specificity of the RT-PCR products was confirmed by analysis of melting curves and by omission of the reverse transcriptase. Human $\beta$-actin gene expression from the same RNA sample was also tested for normalization and quantification. The result was expressed as the fold expression normalized to $\beta$-actin. The gene expression was considered not detectable if the ratio of the gene against $\beta$-actin was smaller than 0.001 . The data of the undetectable gene, was not plotted in Figures 3 and 4.

2.4. Statistical Analyses. For PCR array data analysis we used the SABiosciences $\mathrm{RT}^{2}$ Profiler Data Analysis Software to determine gene expression profiles (http://pcrdataanalysis .sabiosciences.com/pcr/arrayanalysis.php), which determined fold regulation values for each gene using the relative quantification $2-\Delta \Delta \mathrm{Ct}$ method. $\Delta \mathrm{Ct}$ values were normalized using the mean values of three housekeeping genes: $\beta$-Actin, $\beta$-2-microglobulin, and GAPDH. All wells with a Ct value above 35 cycles were excluded from the analysis. This left 84 transcripts for analysis. Mann Whitney tests were used to compare means of continuous variables. Nonparametric test with Spearman's rank correlation coefficient was used for analyzing correlation. A $P$ value $<0.05$ was considered significant. Graphs were generated by GraphPad Prism (GraphPad, Inc., La Jolla, CA).

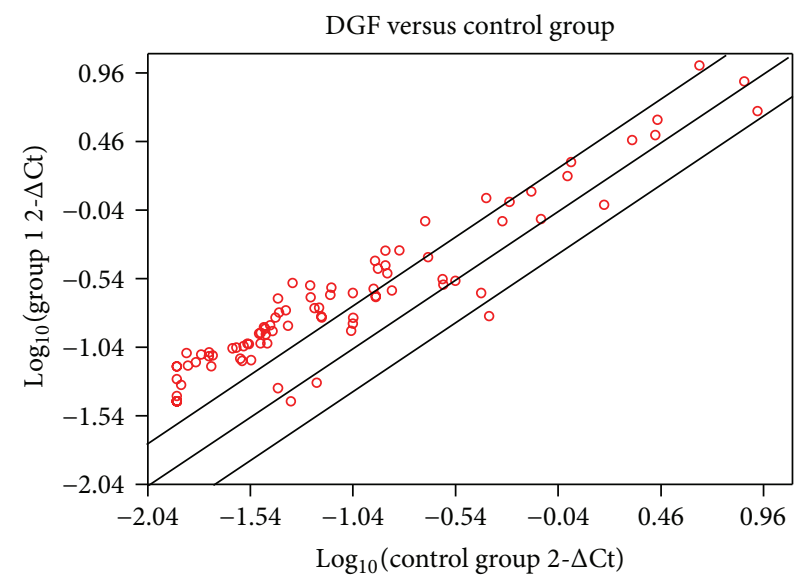

FIGURE 1: Scatter plot analysis of gene expression profiling on expanded criteria of kidney transplant patients. cDNA microarray analysis on 16 RNA samples from kidney transplant patients. After normalization on housekeeping genes, the final scores of each of the genes of all arrays were compared with those from the control array. The scatter plot was acquired as described in Materials and Methods. The $y$-axis represents log scores from DGF patients (group 1) and the $x$-axis represents log scores from no DGF patients (group 2). Each symbol represents one gene. Those gene outside the boundaries represent 2-fold higher or lower expressed in DGF patients.

\section{Results}

3.1. Microarray. Quantitative real-time PCR microarray was used to analyze the gene expression profile in biopsy samples of 16 expanded criteria kidney transplant patients. We analyzed and compared the inflammatory genes profile 


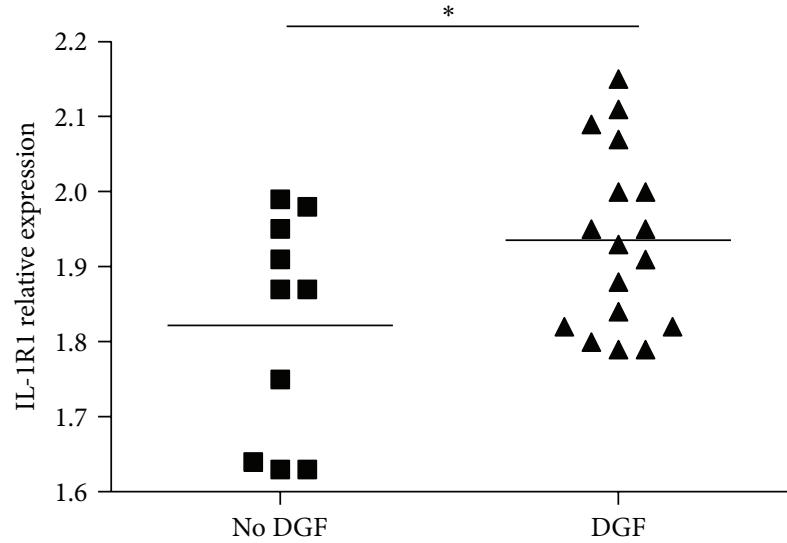

(a)

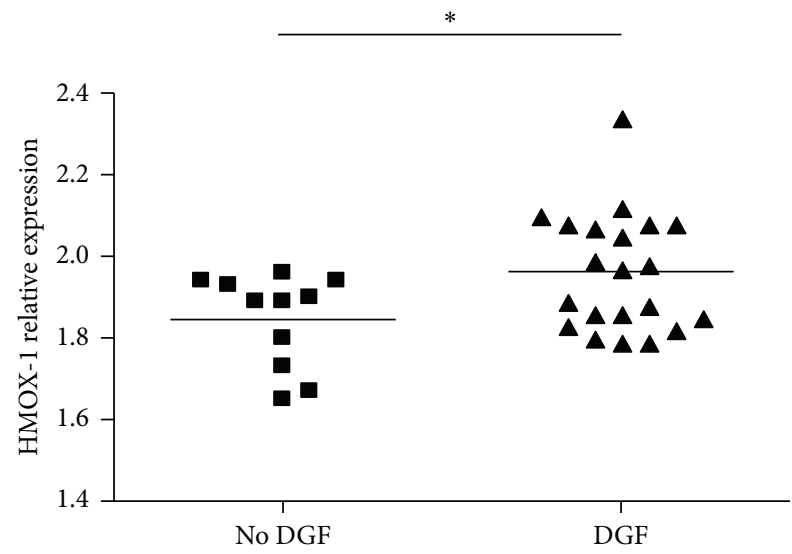

(c)

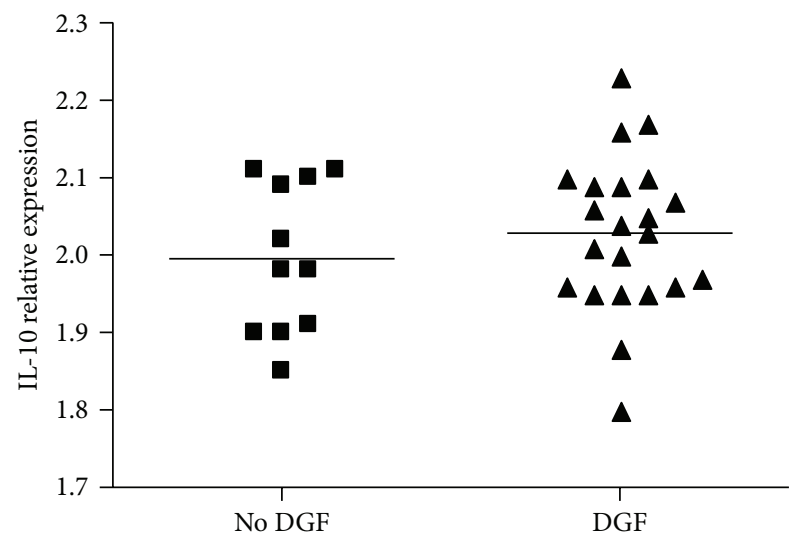

(e)

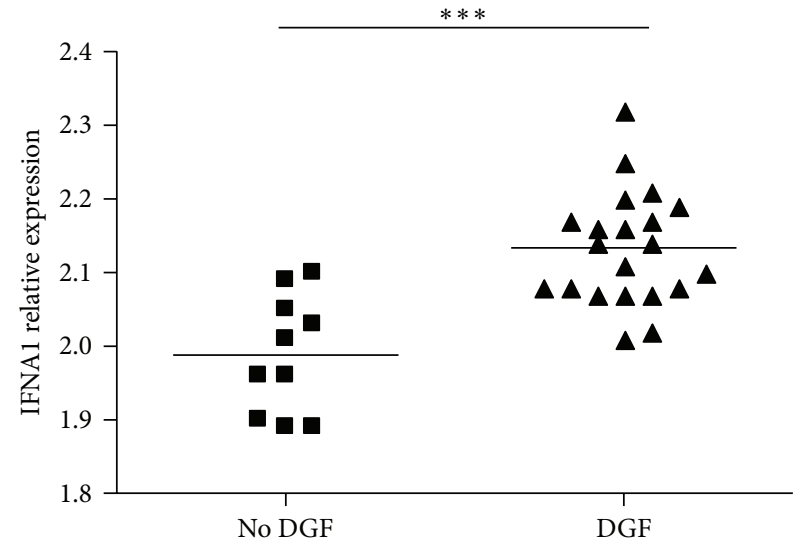

(b)

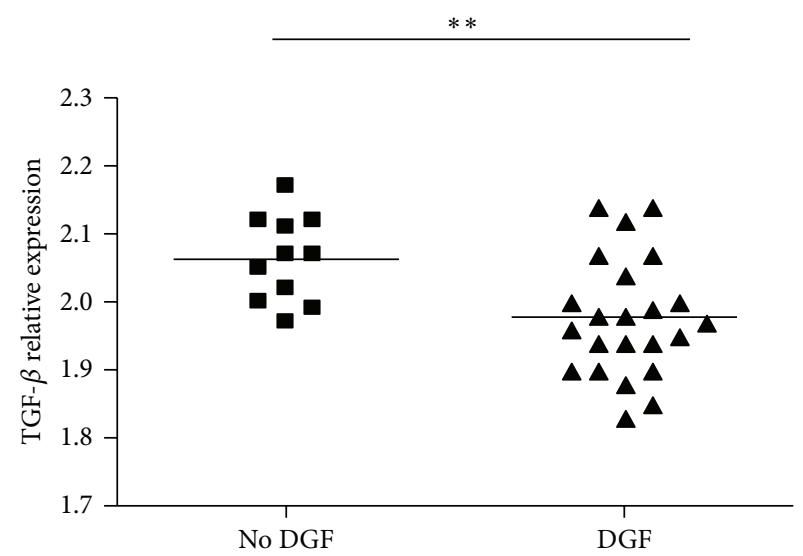

(d)

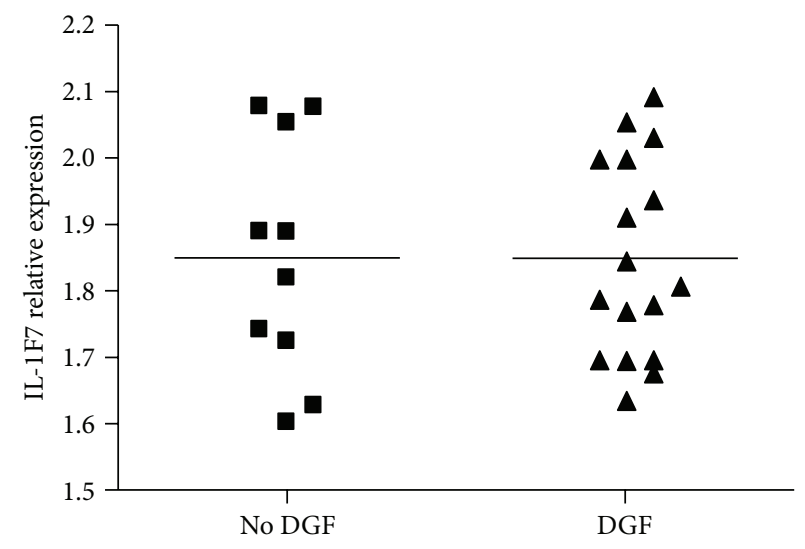

(f)

FIGURE 2: Real-time PCR results evaluating mRNA levels of inflammatory pathway related genes in kidney transplant patients. IL-1R1, IFNA1, HMOX-1, TGF- $\beta$, IL-10, and IL-1F7 were assayed by real-time PCR in whole kidney RNA samples from no DGF patients and DGF patients. Values were normalized to the level of $\beta$-actin RNA. Relative expression levels of all genes were calculated as $2^{\wedge}[(\mathrm{Ct}$ reference gene $)-(\mathrm{Ct}$ target gene) $] .{ }^{*} P<0.05,{ }^{* *} P<0.01$, and ${ }^{* * *} P<0.001$ by Mann Whitney test.

between DGF $(n=8)$ and no DGF $(n=8)$ patients. Supplementary Table 2 shows a summary of the clinical characteristics of the patients enrolled for the microarray study. Seven day posttransplant kidney biopsies samples were used to perform the assay. From a total of 84 genes analyzed, 58 genes were upregulated while only 1 gene was downregulated in kidney biopsies from patients with DGF compared with no DGF (Figure 1). The most relevant genes upregulated, at least by two- or more-fold were IL1R1, IL-10, IFNA1, IL-1F7, and HMOX-1 (Table 3). On the contrary, only TGF- $\beta$ was downmodulated in DGF patients (Table 3). In order to confirm the results obtained with the 


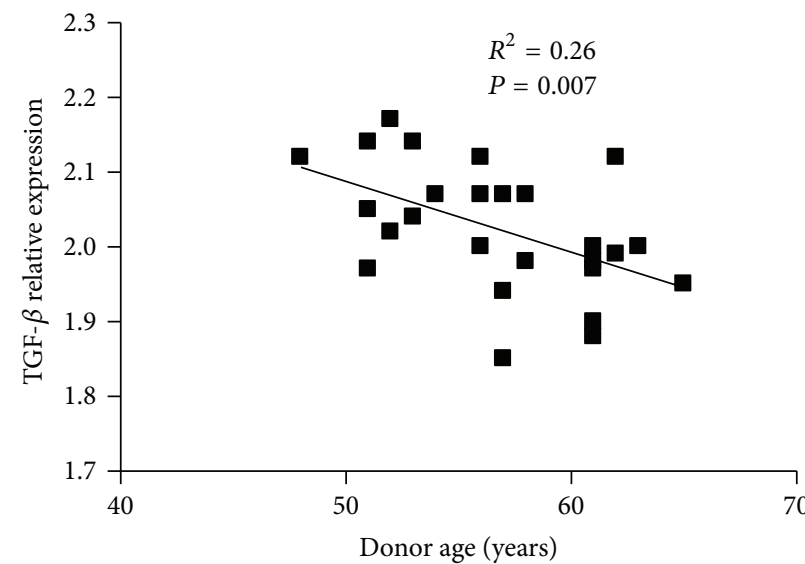

(a)

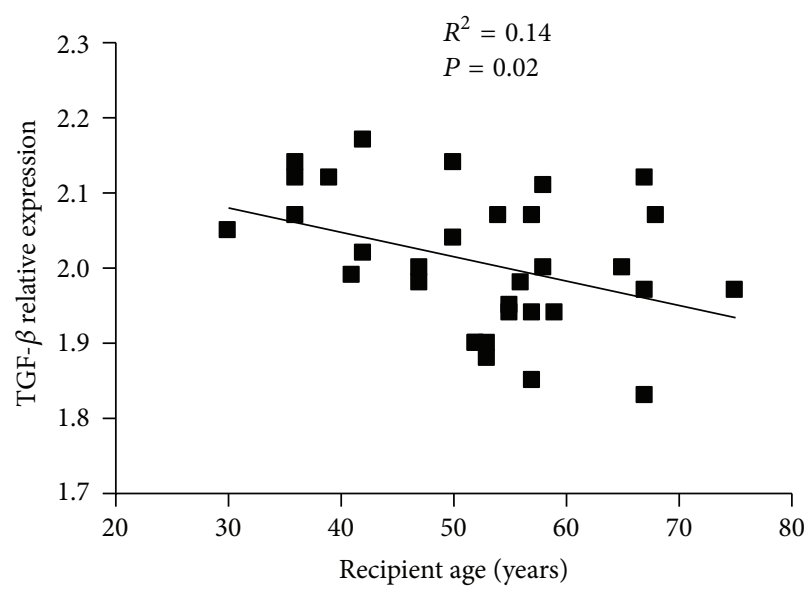

(c)

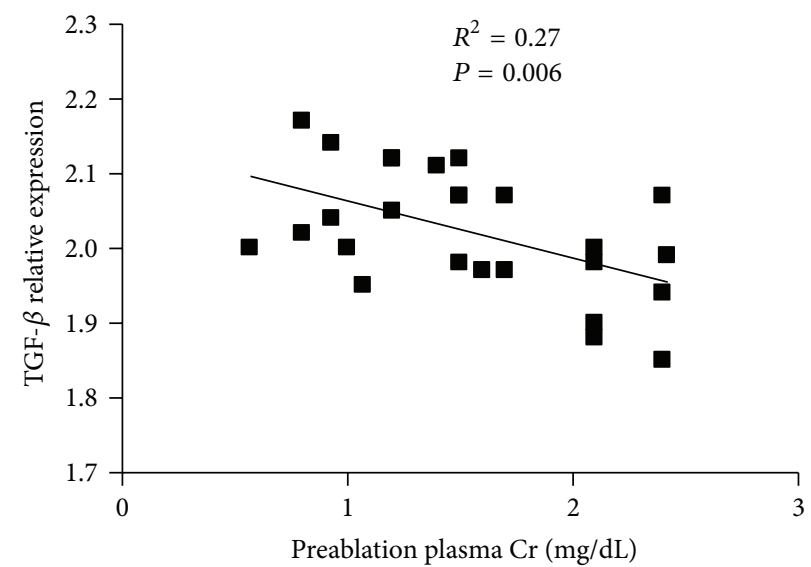

(b)

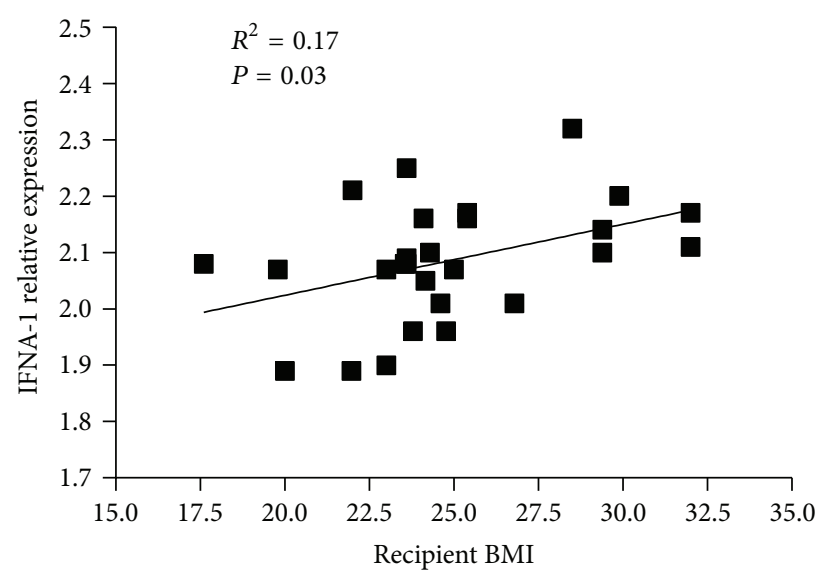

(d)

FIGURE 3: Correlation between the gene expression levels and recipient and donor risk factors. Correlation between donor age and TGF- $\beta$ (a), preablation creatinine and TGF- $\beta$ (b), recipient age and TGF- $\beta$ (c), and recipient BMI and IFNA-1 (d). Regression lines are shown in each figure with correlation coefficients $\left(R^{2}\right)$ and $P$ values.

microarray, we then performed real-time PCR for these genes. For this confirmation assay, we used a total of 34 biopsies samples (11 no DGF and 23 DGF patients). Although we were not able to detect some of the genes in all biopsies samples analyzed, the results obtained with the realtime PCR assay confirmed that IL-1R1 (Figure 2(a)), IFNA1 (Figure 2(b)) and HMOX-1 (Figure 2(c)) genes were upregulated and TGF- $\beta$ (Figure 2(d)) gene was downregulated in DGF patients (Figure $1, P<0.01$ ). However, we were unable to show statistical differences in IL-10 (Figure 2(e)) and IL1F7 (Figure 2(f)) genes between groups of patients analyzed.

3.2. Correlations between Gene Expression and Clinical Features. To further determine if the changes in gene expression observed in the biopsies could be related to donor specific characteristics, CIT, or recipients features we analyzed correlations between genes expression and clinical parameters.
The donor specific characteristics analyzed were the age, preablation creatinine and the body mass index (BMI). Correlations were performed with the genes that were upand downmodulated. We did not find any correlation with donor BMI. However, we found a correlation between TGF- $\beta$ gene expression with donor age (Figure 3(a)) and preablation creatinine (Figure 3(b)). Then, the correlations were analyzed between CIT and genes expression. Although, we did not find statistical significant correlations between this risk factor and inflammatory markers, we found a trend between IFNA1 and CTI $(P=0.057$; data not shown). Following this, the analysis was performed with recipient characteristics, such as age, BMI, and time on dialysis. We did not find correlations with time on dialysis. However, we found that TGF- $\beta$ expression inversely correlated with recipient age (Figure $3(\mathrm{c})$ ). Also, there was a positive correlation between IFNA1 and recipient BMI (Figure 3(d)). Finally, the correlations were examined with markers of kidney function during the first two days 


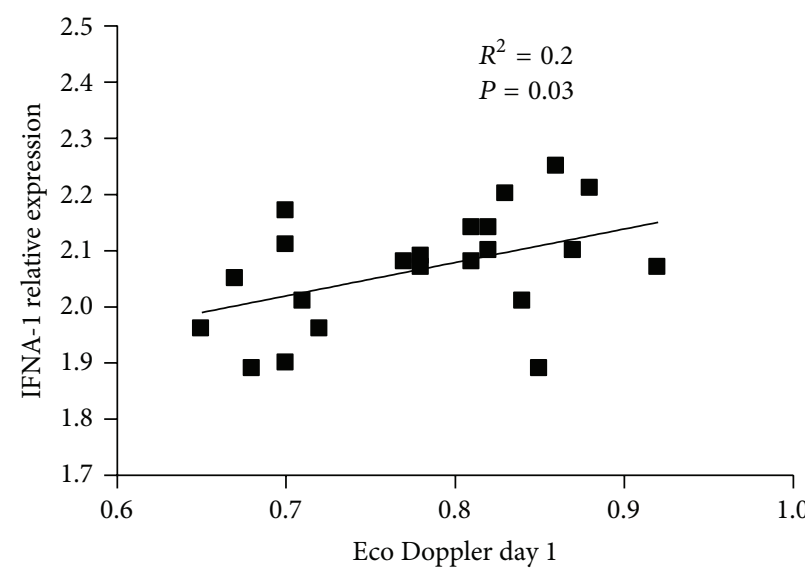

(a)

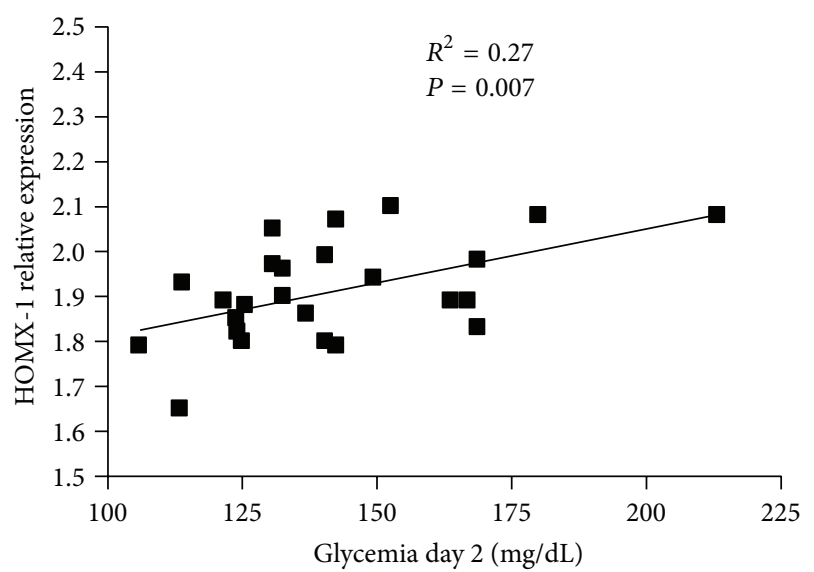

(c)

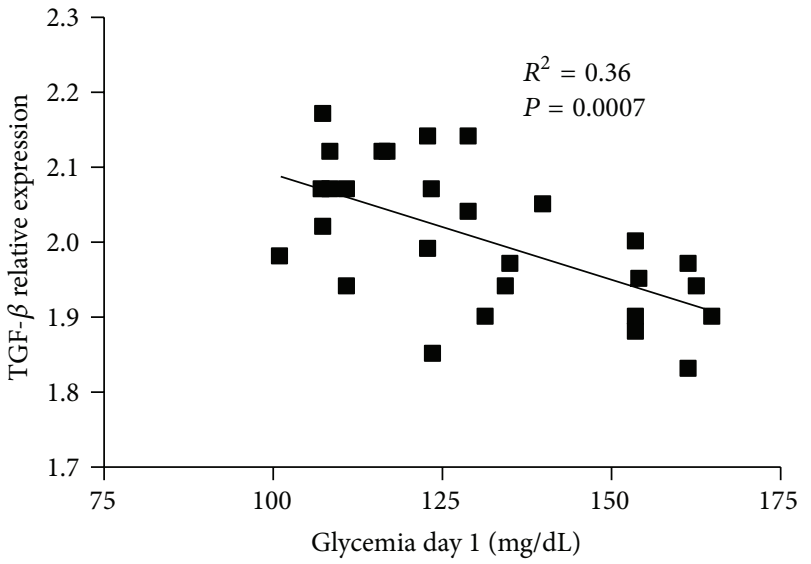

(e)

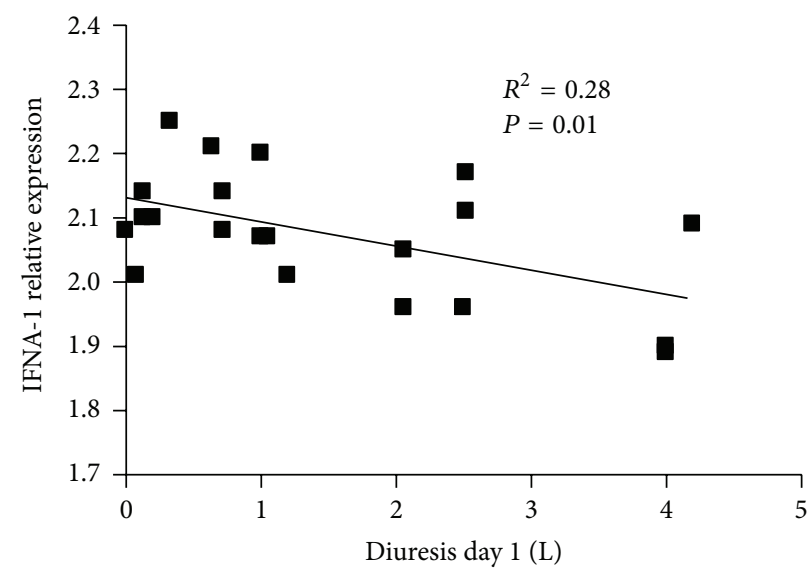

(b)

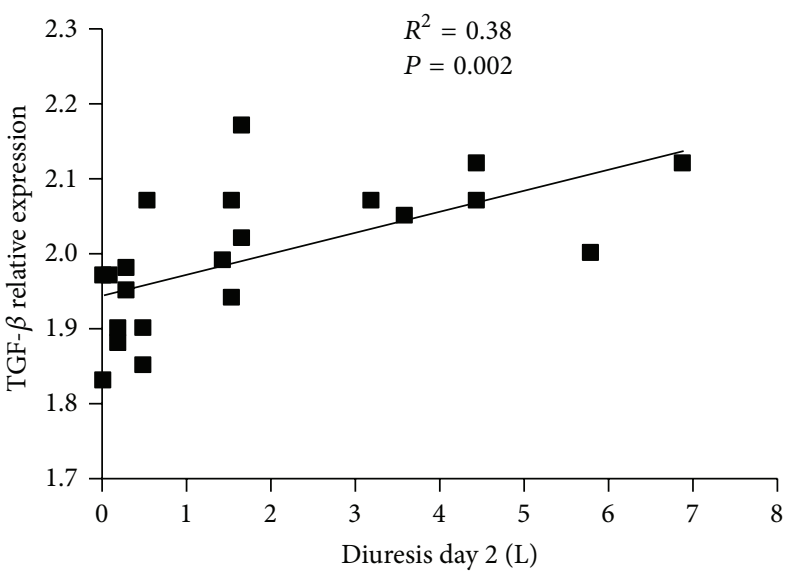

(d)

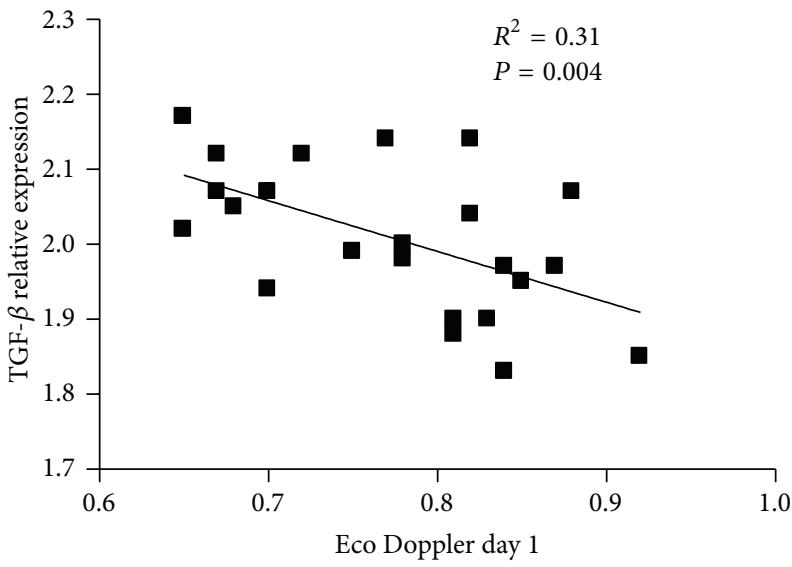

(f)

FIGURE 4: Correlation between the gene expression levels and kidney functional parameters. Correlation between Eco Doppler index of day 1 and IFNA1 (a), diuresis of day 1 and IFNA1 (b), glycemia of day 2 and HMOX-1 (c), diuresis of day 2 and TGF- $\beta$ (d), glycemia of day 1 and TGF- $\beta$ (e), and Eco Doppler index of day 1 and TGF- $\beta$ (f). Regression lines are shown in each figure with correlation coefficients $\left(R^{2}\right)$ and $P$ values.

after the transplant. When IFNA1 was analyzed, a positive and negative correlation was observed with the Doppler ultrasound resistive index and diuresis, respectively (Figures 4(a) and 4(b)). Furthermore, for HMOX-1 a positive correlation was observed with glycemia (Figure 4(c)). Finally, for TGF- $\beta$ we found a positive correlation with diuresis (Figure 4(d)) and the amount of units of insulin administered ( $P=0,001$; data not shown) to the patients, while negative correlation was observed with glycemia (Figure 4(e)) and Doppler ultrasound resistive index (Figure 4(f)). 
TABLE 3: Real-time PCR microarray: genes up- and downmodulated in kidney biopsies from DGF versus no DGF patients.

\begin{tabular}{lcc}
\hline & Mediators & Fold change \\
\hline \multirow{3}{*}{ Upregulation } & IL-1R1 & 4.9 \\
& IL-10 & 4.8 \\
& IFNA1 & 4.4 \\
IL-1F7 & 4.3 \\
Downregulation & HMOX-1 & 2.9 \\
\hline
\end{tabular}

\section{Discussion}

Sometimes a biopsy is performed at the time of transplantation in order to check for preexisting lesions in the donor kidney. However, it has not been helpful as an indicator for therapeutic intervention. Recent findings suggest the importance of recipient factors in addition to the donor factors [5]. In this study we performed a 7 day posttransplant biopsy that should reflect both donor and recipient factors, although the interpretation of the data requires a comprehensive analysis because multiple factors are involved.

A systematic biological assessment of these molecular changes suggests that the inflammatory process driven by ischemia reperfusion injury (IRI) is largely responsible for DGF occurrence. The analysis of the microarray shows that several inflammatory mediators are upregulated in DGF patients. The most significant mediators but not the only ones were IL-R1, IL-10, IFNA1, IL-1F7, and HMOX-1. However, only IFNA1, IL-1R1, and HMOX-1 were confirmed to differ between DGF and no DGF group by real-time PCR. It is important to mention that the partial selection of the mediators analyzed for confirmation with the real-time PCR was chosen based on the fold changes that we found in the microarray.

It is known that drugs used for immunosuppression may affect DGF and gene expression profile [11]. For example, it has been described that sirolimus prolongs recovery from delayed graft function after cadaveric renal transplantation [12]. In our study, biopsies were obtained before the introduction of sirolimus in the immunosuppressive regime. Therefore, gene expression data was not influenced by sirolimus. It is important to mention that in this study, the differential gene expression profile between DGF and no DGF patients was not influenced by immunosuppression drugs, since all patients received the same immunosuppressive regime.

Based on the function of each IFNA1 and IL-1R1, we can speculate that the pattern of cytokines upregulated was compatible with a proinflammatory state. For example, IFNA1 has a well-known antiviral action and also plays a major role in the adaptive immune response acting on dendritic cells, NK cells, and lymphocytes $[13,14]$. Furthermore, IL-1R1 is a signaling receptor for IL-1. This cytokine is a potent factor with pleiotropic functions such as stimulating angiogenesis at inflamed tissue sites, triggering proinflammatory cytokine and contributing to the polarization of Th17 cells [15]. The upregulation of IL-1R1 observed in biopsies of DGF patients suggests that this kidney may be more suitable to respond under inflammatory microenvironments, where the IL-1 is present.

In agreement with this proinflammatory microenvironment, we found that TGF- $\beta$ is downmodulated in DGF patients. TGF- $\beta$ is a $25-\mathrm{kDa}$ homodimeric peptide with pleiotropic activity on different cell types [16]. Their immunosuppressive effects are known by inhibiting lymphocyte activation, but also proinflammatory activity has been described $[16,17]$. Moreover, recent studies suggest that high levels of TGF- $\beta$ activated T cells that cause cytotoxic damage and acute rejection [18]. However, also a low TGF- $\beta$ production in both the donor and recipient was associated with risk for early rejection and worse graft function at 4 years [18].

HMOX-1 is another mediator that is upregulated in DGF patients. HMOX-1 is a very important enzyme which degrades heme into carbon monoxide, biliverdin, and free iron $[19,20]$. The expression of HMOX-1 is inducible in response to pathophysiological stresses and it has antioxidant, anti-inflammatory, and antiapoptotic activity $[19,20]$. In fact, the expression of HMOX-1 protects from the induction of chronic allograft rejection $[21,22]$. Also, it has been described that TGF- $\beta$ induces HMOX-1 [23]. However, since TGF- $\beta$ is downmodulated in our study, we believe that HMOX-1 upregulation is due to the proinflammatory microenvironment. Perhaps, the high levels of HMOX-1 may act as feedback mechanisms that tend to control the proinflammatory state.

Differences between no DGF and DGF groups were also seen in long-term outcome such as patients' survival and graft function (data not shown), as was expected. At three years after kidney transplant, $91 \%$ of the patients of the no DGF group were alive and with a good kidney function; however with the no DGF group 74\% were alive with normal kidney function.

The findings in biopsies taken at seven days are the results of factors contributed by the donor (such as age, $\mathrm{BMI}$, and preablation creatinine), the procurement period (CIT, preservation liquid), the receptor (age, BMI), and early posttransplant period (ischemia reperfusion injury). In fact, there are much more factors that influence the DGF, such as immunosuppression therapy [11]. The relative impact of each of the markers on DGF is difficult to assess. However, by using the inflammatory markers as the endpoint and performing the correlations with each of the factors that contributed to the DGF, we determined the relative strength of each factor on the inflammatory process. Of the donor derived factors analyzed, donor age and preablation creatinine were associated with TGF- $\beta$. Since preablation, creatinine reflects the state of the kidney to be engrafted; this association with TGF- $\beta$ could be expected. Surprisingly, we did not find a significant correlation with CIT as was described in [24], but we did find a trend with IFNA1 ( $P=0,057$; data not shown). Perhaps, the fact that there was not difference in CIT between groups (Table 2) underscores this result. Of the recipient derived factors, the age was associated with TGF- $\beta$, while the BMI with IFNA-1. Thus, recipient factors act both on proand anti-inflammatory mediators. Based on the link between adipose tissue and inflammation [25] we can speculate that obesity influences more over the proinflammatory markers, 
while the recipient age decreases the anti-inflammatory marker. The result of the recipient factors is to shift the balance to a proinflammatory state. However, it seems that the donor factors influence the inflammation by decreasing the immunosuppressive cytokine, such as TGF- $\beta$.

Finally, we found more correlations at the posttransplant period, more precisely with kidney function markers. Some of them may be ascribed to the IRI. For example, the association between IFNA-1 with diuresis and ultrasound Doppler, HMOX-1 with and glycemia, and TGF- $\beta$ with diuresis, ultrasound Doppler, glycemia, and units of insulin administration might represent the sum of the factors derived from the donor and the recipient plus the reperfusion injury.

Gene array has been used before in kidney transplantation $[26,27]$. Most of them were used to monitor the graft status, infection, or graft rejections. Despite the high cost of the technique, by performing the gene array we may detect biomarkers that allow us to predict the outcome of the graft.

\section{Conclusion}

Our results identify inflammatory molecular changes in kidney transplant of DGF patients that associates with clinical risk factors. The strength of these factors on the inflammatory process is uneven. Overall, these results suggest for the first time that changes in some inflammatory mediators in kidney transplantation recipients may be ascribed to donors while others to the recipients' characteristics.

\section{Abbreviations}

BMI: $\quad$ Body mass index

CIT: $\quad$ Cold ischemia time

DGF: Delayed graft function

HMOX-1: Heme oxygenase 1

IFNA1: Interferon alpha 1

IL-1F7: Interleukinl family member 7

IL-1R1: Interleukin 1 receptor, type I

IL-10: Interleukin 10

IRI: Ischemia reperfusion injury

TGF- $\beta$ : Transforming growth factor Beta.

\section{Conflict of Interests}

The authors declare that there is no conflict of interests regarding the publication of this paper.

\section{Authors' Contribution}

Casadei Domingo and Incardona Claudio have contributed equally to this work.

\section{Acknowledgments}

The excellent secretarial works of Ms. Soledad Basualdo are gratefully acknowledged. This work was partially supported by Grants UBACYT 940, CONICET1001, PICT2331, and Fundación GADOR to H.E.C.

\section{References}

[1] S. G. Yarlagadda, S. G. Coca, R. N. Formica, E. D. Poggio, and C. R. Parikh, "Association between delayed graft function and allograft and patient survival: a systematic review and metaanalysis," Nephrology Dialysis Transplantation, vol. 24, no. 3, pp. 1039-1047, 2009.

[2] S. G. Yarlagadda, S. G. Coca, A. X. Garg et al., "Marked variation in the definition and diagnosis of delayed graft function: a systematic review," Nephrology Dialysis Transplantation, vol. 23, no. 9, pp. 2995-3003, 2008.

[3] UNOS, 2010 Annual Data Report, UNOS, 2010.

[4] E. J. M. Bronzatto, K. R. da Silva Quadros, R. L. S. Santos, G. Alves-Filho, and M. Mazzali, "Delayed graft function in renal transplant recipients: risk factors and impact on 1-year graft function: a single center analysis," Transplantation Proceedings, vol. 41, no. 3, pp. 849-851, 2009.

[5] A. Siedlecki, W. Irish, and D. C. Brennan, "Delayed graft function in the kidney transplant," American Journal of Transplantation, vol. 11, no. 11, pp. 2279-2296, 2011.

[6] A. J. Turunen, L. Lindgren, K. T. Salmela, L. E. Kyllönen, J. Petäjä, and E. J. Pesonen, "Intragraft coagulation events and delayed graft function in clinical renal transplantation," Transplantation, vol. 85, no. 5, pp. 693-699, 2008.

[7] A. J. Turunen, L. Lindgren, K. T. Salmela et al., "Association of graft neutrophil sequestration with delayed graft function in clinical renal trasplantation," Transplantation, vol. 77, no. 12, pp. 1821-1826, 2004.

[8] P. Hribova, J. Lacha, K. Kotsch et al., "Intrarenal cytokine and chemokine gene expression and kidney graft outcome," Kidney and Blood Pressure Research, vol. 30, no. 5, pp. 273-282, 2007.

[9] A. Figueiredo, P. Moreira, B. Parada et al., "Risk factors for delayed renal graft function and their impact on renal transplantation outcome," Transplantation Proceedings, vol. 39, no. 8, pp. 2473-2475, 2007.

[10] P. Moreira, H. Sá, A. Figueiredo, and A. Mota, "Delayed renal graft function: risk factors and impact on the outcome of transplantation," Transplantation Proceedings, vol. 43, no. 1, pp. 100-105, 2011.

[11] J. Boletis, A. Balitsari, V. Filiopoulos et al., "Delayed renal graft function: the influence of immunosuppression," Transplantation Proceedings, vol. 37, no. 5, pp. 2054-2059, 2005.

[12] R. A. McTaggart, D. Gottlieb, J. Brooks et al., "Sirolimus prolongs recovery from delayed graft function after cadaveric renal transplantation," American Journal of Transplantation, vol. 3, no. 4, pp. 416-423, 2003.

[13] G. Trinchieri, “Type I interferon: friend or foe?" Journal of Experimental Medicine, vol. 207, no. 10, pp. 2053-2063, 2010.

[14] L. C. Platanias, "Mechanisms of type-I- and type-II-interferonmediated signalling," Nature Reviews Immunology, vol. 5, no. 5, pp. 375-386, 2005.

[15] C. A. Dinarello, "A clinical perspective of IL-1 $\beta$ as the gatekeeper of inflammation," European Journal of Immunology, vol. 41, no. 5, pp. 1203-1217, 2011.

[16] F. S. Regateiro, D. Howie, S. P. Cobbold, and H. Waldmann, "TGF-beta in transplantation tolerance," Current Opinion in Immunology, vol. 23, no. 5, pp. 660-669, 2011.

[17] A. Yoshimura and G. Muto, "TGF-beta function in immune suppression," Current Topics in Microbiology and Immunology, vol. 350, pp. 127-147, 2011. 
[18] P. Pribylova-Hribova, K. Kotsch, A. Lodererova et al., “TGF- $\beta 1$ mRNA upregulation influences chronic renal allograft dysfunction," Kidney International, vol. 69, no. 10, pp. 1872-1879, 2006.

[19] M. P. Soares, I. Marguti, A. Cunha, and R. Larsen, "Immunoregulatory effects of HO-1: how does it work?" Current Opinion in Pharmacology, vol. 9, no. 4, pp. 482-489, 2009.

[20] V. Koliaraki and G. Kollias, "A new role for myeloid HO-1 in the innate to adaptive crosstalk and immune homeostasis," Advances in Experimental Medicine and Biology, vol. 780, pp. 101-111, 2011.

[21] N. O. S. Camara and M. P. Soares, "Heme oxygenase-1 (HO-1), a protective gene that prevents chronic graft dysfunction," Free Radical Biology and Medicine, vol. 38, no. 4, pp. 426-435, 2005.

[22] A. E. Courtney and A. P. Maxwell, "Heme oxygenase 1: does it have a role in renal cytoprotection?" American Journal of Kidney Diseases, vol. 51, no. 4, pp. 678-690, 2008.

[23] W. Ning, R. Song, C. Li et al., “TGF- $\beta 1$ stimulates HO-1 via the p38 mitogen-activated protein kinase in A549 pulmonary epithelial cells," American Journal of Physiology-Lung Cellular and Molecular Physiology, vol. 283, no. 5, pp. L1094-L1102, 2002.

[24] L. K. Kayler, T. R. Srinivas, and J. D. Schold, "Influence of CIT-induced DGF on kidney transplant outcomes," American Journal of Transplantation, vol. 11, no. 12, pp. 2657-2664, 2011.

[25] F. P. de Heredia, S. Gomez-Martinez, and A. Marcos, "Obesity, inflammation and the immune system," Proceedings of the Nutrition Society, vol. 71, no. 2, pp. 332-338, 2012.

[26] S. M. Flechner, S. M. Kurian, S. R. Head et al., "Kidney transplant rejection and tissue injury by gene profiling of biopsies and peripheral blood lymphocytes," American Journal of Transplantation, vol. 4, no. 9, pp. 1475-1489, 2004.

[27] K. S. Famulski, D. G. de Freitas, C. Kreepala et al., "Molecular phenotypes of acute kidney injury in kidney transplants," Journal of the American Society of Nephrology, vol. 23, no. 5, pp. 948-958, 2012. 


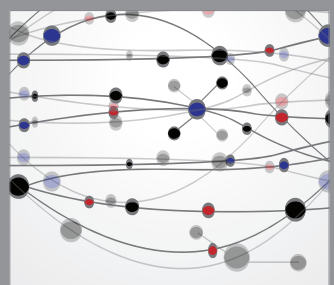

The Scientific World Journal
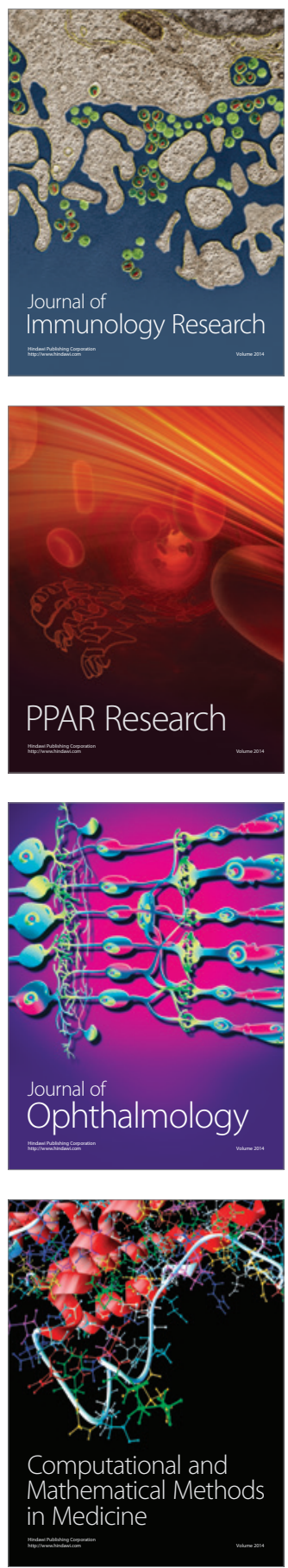

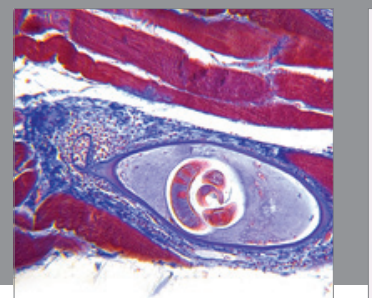

Gastroenterology

Research and Practice
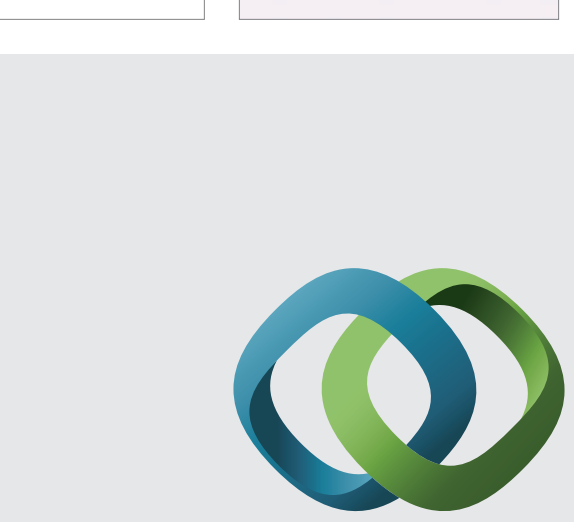

\section{Hindawi}

Submit your manuscripts at

http://www.hindawi.com
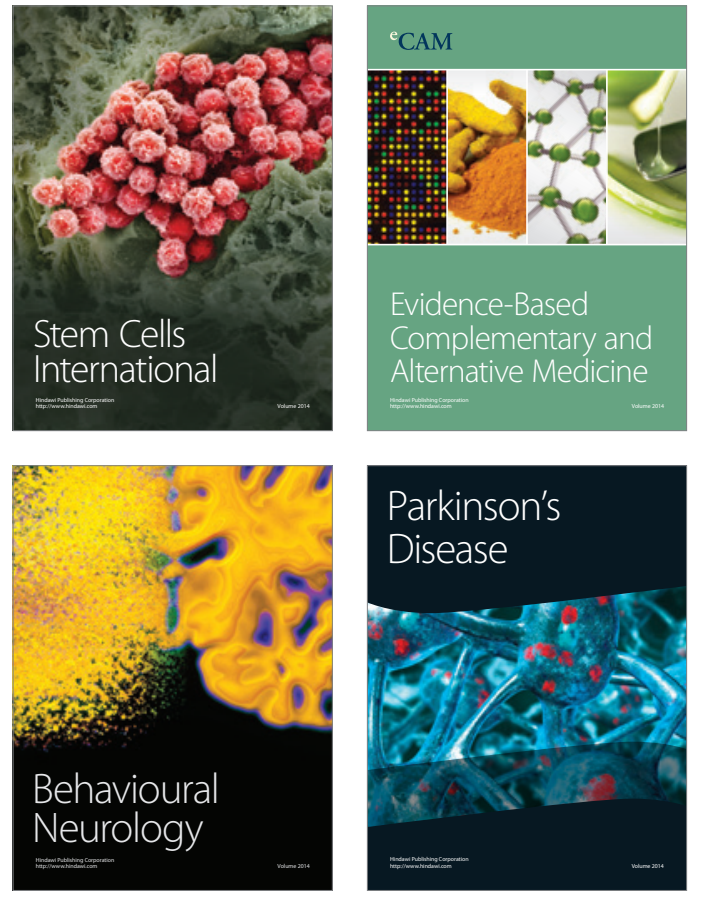
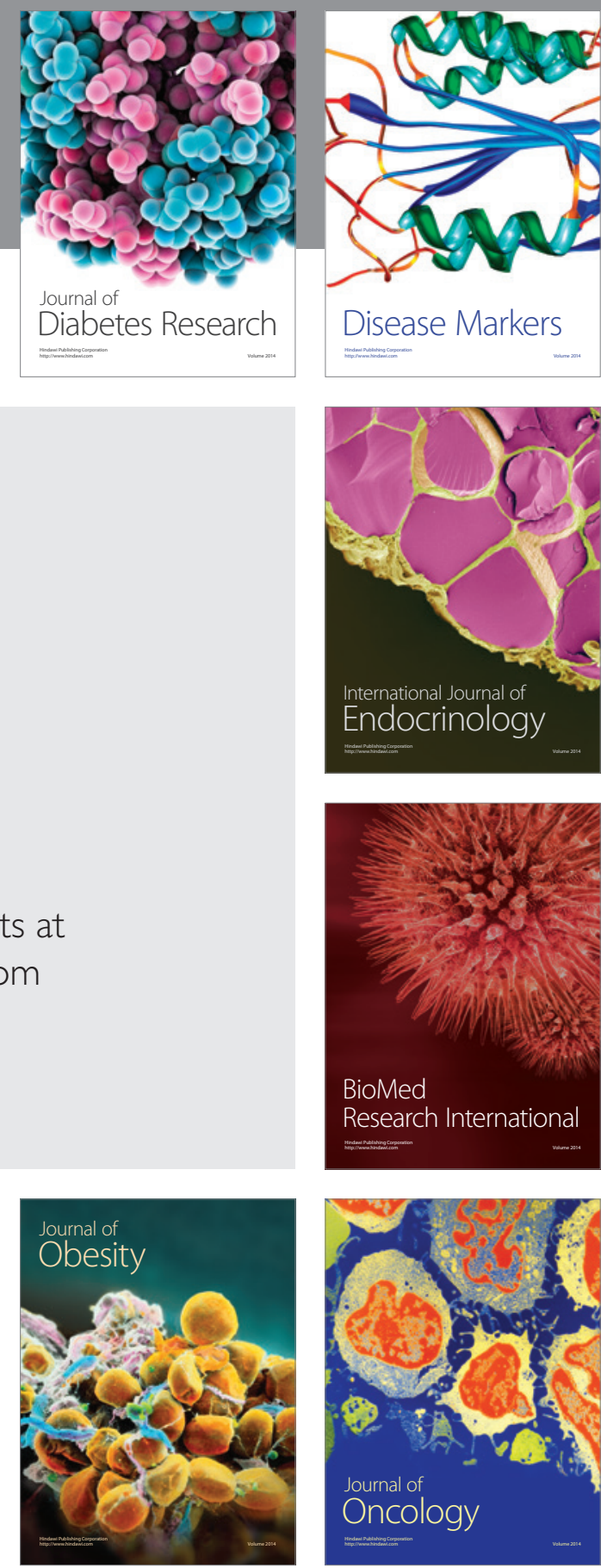

Disease Markers
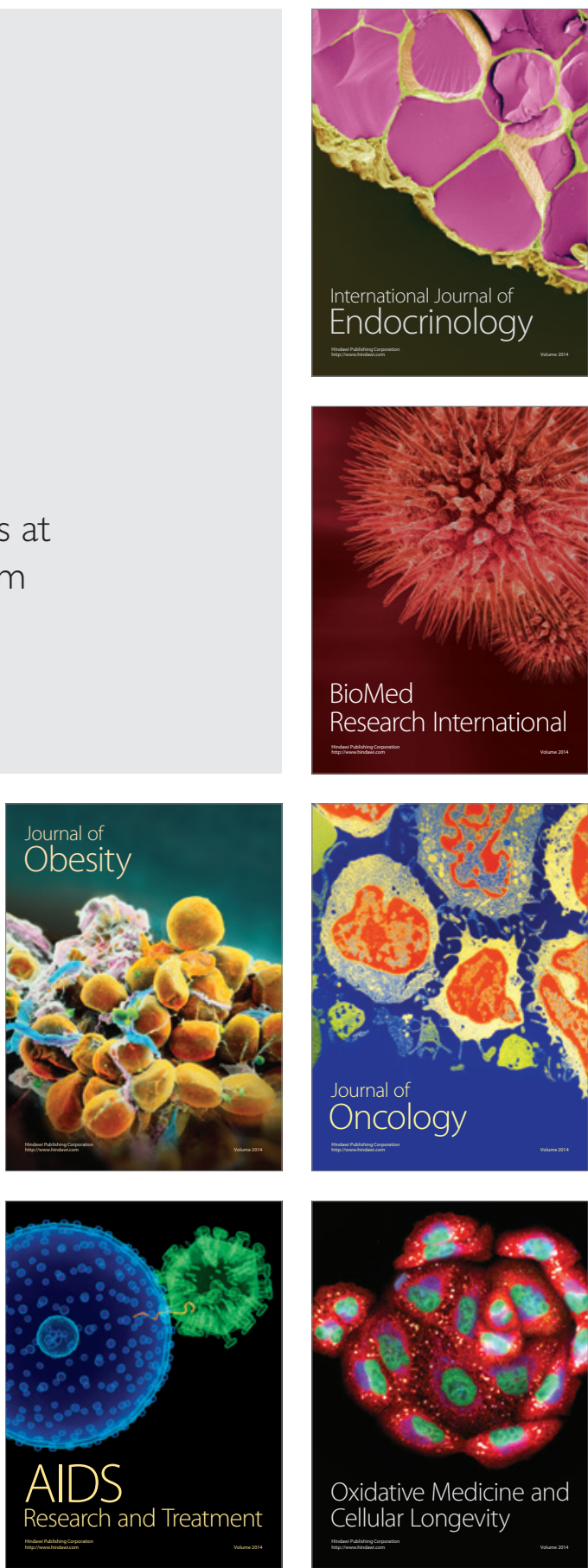